\title{
Possible Association of the Ubiquitin-Specific Peptidase 46 Gene (USP46) with Affective Temperamental Traits in Healthy Korean Volunteers
}

\author{
Young Jun Boo ${ }^{1,2}$, Chun II Park ${ }^{1,2}$, Hae Won $\mathrm{Kim}^{2,3}$, Se Joo Kim ${ }^{1,2} \bowtie$, and Jee In Kang ${ }^{1,2} \bowtie$ \\ ${ }^{1}$ Department of Psychiatry, Yonsei University College of Medicine, Seoul, Republic of Korea \\ ${ }^{2}$ Institute of Behavioral Science in Medicine, Yonsei University College of Medicine, Seoul, Republic of Korea \\ ${ }^{3}$ Department of Medical Education, Yonsei University College of Medicine, Seoul, Republic of Korea
}

Objective Ubiquitin-specific peptidase 46 gene (USP46) polymorphisms is part of ubiquitin-proteasome system, which is responsible for dynamic cellular processes such as the regulation of cell cycle. USP46 has been reported to be associated with major depressive disorder. The objective of the present study was to investigate the association of USP46 polymorphisms with affective temperamental traits in healthy subjects.

Methods A total of 557 Korean healthy volunteers were recruited, and 545 subjects ( 328 male, 217 female) were included in the final analysis. The DNA of the subjects was isolated from saliva samples. Two single-nucleotide polymorphisms (SNPs) rs346005, rs2244291 in USP46 were genotyped. Affective temperaments were assessed using the Korean version of Temperament Evaluation of the Memphis, Pisa, Paris, and San Diego Autoquestionnaire (TEMPS-A).

Results A significant association was found between rs346005 genotypes and TEMPS-A only in male subjects. In particular, subjects with the CC genotype of rs346005 showed a more depressive temperament than subjects with AA or CA genotypes in males. For rs2244291, there were no associations between the rs2244291 genotypes and TEMPS-A scores.

Conclusion Some affective temperaments may serve as a genetic predisposing factors for affective disorders, such as depressive disorder, via vulnerability genes related to the ubiquitin-proteasome system.

Psychiatry Investig 2019;16(1):87-92

Key Words Affective temperament, Genetic association study, TEMPS-A, Ubiquitin-proteasome system, USP46.

\section{INTRODUCTION}

Affective temperaments refer to characteristic patterns in the quality and intensity of baseline mood levels and emotional and behavioral responses and regulations to internal and external stimuli, which tend to appear early in life and be

Received: April 30, 2018 Revised: August 14, 2018 Accepted: October 2, 2018

$\triangle$ Correspondence: Se Joo Kim, MD, PhD

Department of Psychiatry and Institute of Behavioral Science in Medicine, Yonsei University College of Medicine, 50-1 Yonsei-ro, Seodaemun-gu, Seoul 03722, Republic of Korea

Tel: +82-2-2228-1620, Fax: +82-2-313-0890, E-mail: kimsejoo@yuhs.ac

$\bowtie$ Correspondence: Jee In Kang, MD, PhD

Department of Psychiatry and Institute of Behavioral Science in Medicine, Yonsei University College of Medicine, 50-1 Yonsei-ro, Seodaemun-gu, Seoul 03722, Republic of Korea

Tel: +82-2-2228-1620, Fax: +82-2-313-0890, E-mail: jeeinkang@yuhs.ac

(c) This is an Open Access article distributed under the terms of the Creative Commons Attribution Non-Commercial License (https://creativecommons.org/licenses/bync/4.0) which permits unrestricted non-commercial use, distribution, and reproduction in any medium, provided the original work is properly cited. temporally stable. ${ }^{1-3}$ Akiskal et al. ${ }^{1}$ proposed five affective temperamental traits: depressive, cyclothymic, hyperthymic, irritable, and anxious temperaments and developed a valid assessment scale for affective temperaments, viz. the Temperament Evaluation of the Memphis, Pisa, Paris, and San Diego Autoquestionnaire (TEMPS-A). These affective temperaments are not only considered to be subclinical manifestation of affective disorders, ${ }^{1,4,5}$ but are also known to play a crucial role in the clinical prognosis of psychiatric illnesses. ${ }^{6-9}$ A recent study of affective temperament and suicide showed that an anxious temperament is a strong risk factor for suicidal attempts, regardless of the presence of mental disorders, while depressive and irritable temperaments are risk factors for suicidal attempts in patients with mental disorders. ${ }^{6}$ Therefore, a better understanding of affective temperaments is crucial to the understanding of the development of and treatments for mental disorders.

Affective temperaments are known to be moderately heri- 
table, with heritability ranging from $21 \%$ to $52 \%$ for the five affective temperaments in 101 bipolar families. ${ }^{10}$ Additionally, several candidate gene association studies have been conducted to identify genes for affective temperaments. Previous genetic association approaches of affective temperaments usually targeted monoaminergic systems, including serotoninrelated genes, ${ }^{11-14}$ and dopaminergic genes. ${ }^{13}$ A genome-wide association study has revealed several variants that are significantly associated with a specific affective temperament in bipolar patients. ${ }^{15}$ Since affective temperaments may be quantitative endophenotypes for affective disorders, representing genetic heterogeneity, the interest in genetic studies of affective temperaments has increased.

Besides the monoaminergic genes, the ubiquitin-proteasome system (UPS), a major protein degradation pathway, which is responsible for dynamic cellular processes, such as the regulation of cell cycle and the response to genotoxic and proteotoxic stress, ${ }^{16}$ may be a potential candidate system contributing to affective temperaments as well as affective disorders. The UPS is a critical contributor to the construction of brain networks via extensive regulation of synaptic plasticity. ${ }^{17,18}$ In recent years, synaptic plasticity has emerged as a component of the pathophysiology of depression. ${ }^{19,20}$ Interestingly, some studies have revealed direct associations between the UPS and depressive disorders. ${ }^{21-23}$ In an animal study using quantitative trait locus mapping, the ubiquitin-specific peptidase 46 gene (USP46) was proposed to be responsible for depressive-like behavior in mice in depression-inducing tests, such as tail suspension and forced swimming tests. ${ }^{23}$ Furthermore, USP46 knock-out mice also showed differences in depression-inducing test. ${ }^{22}$ Moreover, a human study showed significant differences in USP46 single-nucleotide polymorphisms (SNPs) between patients with depressive disorder and normal controls. ${ }^{21}$ Taken together, these findings may indicate that USP46 may be a candidate gene involved in the development of affective temperaments, particularly in depressive temperaments, as a subclinical manifestation of affective disorders.

The objective of the present study was to investigate the association of USP46 polymorphisms with affective temperamental traits in healthy subjects.

\section{METHODS}

\section{Subjects}

A total of 557 healthy Korean volunteers (334 males, 223 females) were recruited using advertisements. Most of the subjects were college students. All participants were interviewed by psychiatrists, and subjects with any current or previous Axis I psychiatric disorders according to the Diagnostic and Statistical Manual of Mental Disorders, 4th Edition (DSM-IV), a family history of psychiatric disorders, or neurologic disorders were excluded. Twelve subjects (2.16\% of the total subjects) were excluded based on the exclusion criteria. A total of 545 subjects (328 male, 217 female) were included in the final analysis. All subjects provided informed consent before the study. The study protocol was approved by the Institutional Review Board of Severance Hospital (Severance IRB 4-2010-0577).

\section{SNP selection}

To date, only one study has reported positive association between USP46 gene and psychiatric phenotypes. Fukuo et al. ${ }^{21}$ found significant association between haplotypes consisting of three (rs2244291-rs10034164-rs346005) or four (rs12646800rs2244291-rs10034164-rs346005) SNPs of seven tagging SNPs $\left(r^{2} \geq 0.8\right)$ capturing 30 SNPs (minor allele frequency; MAF $\geq 5$ ) covering the USP46 gene region. ${ }^{21}$ Therefore, we selected SNPs of rs12646800, rs2244291, rs10034164, and rs346005 in this study. However, we further excluded rs12646800 and rs10034164, as MAFs were less than 0.1 (0.056 for rs12646800, 0.087 for rs10034164). Especially, there were no minor homozygotes for rs10034164 in the HapMap database (release \#27, population: Japanese in Tokyo). Finally, we included two SNPs (rs2244291 and rs346005) in this study. SNP rs2244291 is located in intron, and SNP rs346005 is located $2 \mathrm{~kb}$ upstream of USP46 start site (USP46; 52590960-52659335, rs346005; 52588572).

\section{Genotyping}

The DNA of the subjects was isolated from saliva collected in OG-500 Oragene ${ }^{\circledR}$ self-collection kit (tube format “OG-500"), using standard techniques. Genotyping of USP46 SNPs was performed using a single-base primer extension assay (ABI PRISM $^{\circledR}$ SNaPShot $^{\mathrm{TM}}$ Multiplex kit; ABI, Foster City, CA, USA) according to the manufacturer's instructions. Genotyping was performed by DNA Link, Inc. (Seoul, South Korea), in a manner blinded to any other study data.

\section{Assessments}

Affective temperament was assessed with the Korean version of the Memphis, Pisa, Paris, and San Diego Autoquestionnaire (TEMPS-A, a self-reported temperament scale for evaluating temperamental dimensions, with five subscales, including depressive, cyclothymic, hyperthymic, irritable, and anxious temperaments. ${ }^{1}$ This scale consists of 110 "yes-or-no" type questions in total, of which 109 are applicable to male subjects. All participants completed the questionnaire.

\section{Statistical analyses}

Previous combined analysis showed the differences in TEMPSA subscales scores between males and females. ${ }^{24}$ Therefore, all the analyses in this study were conducted separately by 
gender. Multiple analyses of covariance (MANCOVA) were performed using the total scores for each TEMPS-A affective temperament dimension as dependent variables, and USP46 SNP genotypes as fixed factors. Age was controlled as a covariate to control possible effects. The two SNPs were analyzed separately for each gender. Further analyses were conducted for significant associations, with Fisher's least significant difference post-hoc tests to evaluate differences among genotypes. Data analysis was performed using SPSS 23.0 for Windows (IBM Corp., Armonk, NY, USA). The statistical significance was set at $\mathrm{p}<0.05$ for all tests.

Table 1. Demographic characteristics of the subjects

\begin{tabular}{lccr}
\hline & Male $(\mathrm{N}=328)$ & Female $(\mathrm{N}=217)$ & \multicolumn{1}{c}{$\mathrm{p}$} \\
\hline Age (years) & $22.55(2.64)$ & $21.57(2.47)$ & $<0.001$ \\
Education (years) & $14.32(1.46)$ & $14.39(1.58)$ & 0.596 \\
TEMPS-A & & & \\
$\quad$ Depressive & $6.73(3.03)$ & $7.51(2.90)$ & 0.003 \\
Cyclothymic & $6.83(4.56)$ & $8.03(4.57)$ & 0.003 \\
Hyperthymic & $11.54(4.79)$ & $10.79(4.54)$ & 0.068 \\
Irritable & $3.60(3.27)$ & $3.73(3.45)$ & 0.633 \\
Anxious & $5.56(5.19)$ & $7.28(5.42)$ & $<0.001$ \\
\hline
\end{tabular}

Mean (SD). SD: standard deviation, TEMPS-A: Temperament Evaluation of the Memphis, Pisa, Paris, and San Diego Autoquestionnaire

\section{RESULTS}

\section{Baseline characteristics}

Baseline demographic data and characteristics, including TEMPS-A scores, are summarized in Table 1. The mean age of the participants was 22.55 years (standard deviation; $\mathrm{SD}=2.64$ years) for males and 21.57 years ( $\mathrm{SD}=2.47$ years) for females. The mean education level of the participants were 14.32 years ( $\mathrm{SD}=1.46$ years) for males and 14.39 years $(\mathrm{SD}=1.58$ years) for females. There were significant differences in age $(\mathrm{p}<0.001)$ and depressive $(\mathrm{p}=0.003)$, cyclothymic $(\mathrm{p}=0.003)$, and anxious $(\mathrm{p}<0.001)$ dimension scores between male subjects and female subjects.

\section{Association analyses between USP46 SNPs and five affective temperamental dimensions}

The genotype distribution of the two USP46 SNPs, rs346005 and rs2244291 were in accordance with the Hardy-Weinberg equilibrium $\left(\chi^{2}=0.068, \mathrm{p}=0.794\right.$ for $\mathrm{rs} 346005, \chi^{2}=0.161, \mathrm{p}=0.6835$ for rs2244291).

MANCOVA results are presented in Table 2 through Table 3. The MANCOVA showed a significant association between TEMPS-A and rs346005 genotypes in male subjects (Wilks $\left.\lambda=0.929, \mathrm{~F}_{(10,640)}=2.384, \mathrm{p}=0.009\right)$. Among temperament dimensions, there was a significant difference in depressive temperament among the genotypes of rs346005 in male sub-

Table 2. Multivariate analysis comparing scores for each affective temperament dimension for USP46 single-nucleotide polymorphism rs346005 (mean \pm SEM) after controlling for age

\begin{tabular}{|c|c|c|c|c|c|c|c|c|}
\hline & \multicolumn{3}{|c|}{ Male $(\mathrm{N}=328)$} & \multirow{2}{*}{$\mathrm{p}$} & \multicolumn{3}{|c|}{ Female $(\mathrm{N}=217)$} & \multirow{2}{*}{ p } \\
\hline & AA $(\mathrm{N}=97)$ & $\mathrm{CA}(\mathrm{N}=161)$ & $\mathrm{CC}(\mathrm{N}=70)$ & & AA $(N=65)$ & $\mathrm{CA}(\mathrm{N}=111)$ & $\mathrm{CC}(\mathrm{N}=41)$ & \\
\hline MANCOVA & \multicolumn{3}{|c|}{ Wilks $\lambda=0.929, \mathrm{~F}_{(10,640)}=2.384, \mathrm{p}=0.009$} & \multicolumn{5}{|c|}{ Wilks $\lambda=0.979, \mathrm{~F}_{(10,418)}=0.450, \mathrm{p}=0.921$} \\
\hline Depressive & $6.79 \pm 0.30$ & $6.20 \pm 0.23$ & $7.87 \pm 0.36$ & $<0.001$ & $7.68 \pm 0.36$ & $7.28 \pm 0.28$ & $7.86 \pm 0.45$ & 0.472 \\
\hline Cyclothymic & $6.47 \pm 0.46$ & $6.78 \pm 0.36$ & $7.45 \pm 0.54$ & 0.380 & $8.34 \pm 0.58$ & $7.96 \pm 0.44$ & $7.71 \pm 0.72$ & 0.886 \\
\hline Hyperthymic & $11.46 \pm 0.48$ & $11.91 \pm 0.38$ & $10.80 \pm 0.57$ & 0.258 & $10.55 \pm 0.57$ & $10.93 \pm 0.44$ & $10.78 \pm 0.71$ & 0.869 \\
\hline Irritable & $3.34 \pm 0.33$ & $3.43 \pm 0.26$ & $4.34 \pm 0.39$ & 0.097 & $3.59 \pm 0.44$ & $3.69 \pm 0.33$ & $4.10 \pm 0.54$ & 0.750 \\
\hline Anxious & $5.97 \pm 0.53$ & $4.97 \pm 0.41$ & $6.36 \pm 0.62$ & 0.120 & $7.53 \pm 0.68$ & $7.16 \pm 0.52$ & $7.22 \pm 0.85$ & 0.906 \\
\hline
\end{tabular}

SEM: standard error of mean, MANCOVA: multiple analyses of covariance

Table 3. Multivariate analysis comparing scores for each affective temperament dimension for USP46 gene single-nucleotide polymorphism rs2244291 (mean \pm SEM) after controlling for age

\begin{tabular}{|c|c|c|c|c|c|c|c|c|}
\hline & \multicolumn{3}{|c|}{ Male $(\mathrm{N}=328)$} & \multirow{2}{*}{$\mathrm{p}$} & \multicolumn{3}{|c|}{ Female $(\mathrm{N}=217)$} & \multirow{2}{*}{$\mathrm{p}$} \\
\hline & AA $(\mathrm{N}=198)$ & GA $(\mathrm{N}=118)$ & $\mathrm{GG}(\mathrm{N}=12)$ & & $\mathrm{AA}(\mathrm{N}=138)$ & GA $(\mathrm{N}=68)$ & $\mathrm{GG}(\mathrm{N}=11)$ & \\
\hline MANCOVA & \multicolumn{3}{|c|}{ Wilks $\lambda=0.966, \mathrm{~F}_{(10,640)}=1.124, \mathrm{p}=0.341$} & \multicolumn{5}{|c|}{ Wilks $\lambda=0.935, \mathrm{~F}_{(10,418)}=1.421, \mathrm{p}=0.168$} \\
\hline Depressive & $6.62 \pm 0.22$ & $6.94 \pm 0.28$ & $6.44 \pm 0.88$ & 0.643 & $7.56 \pm 0.25$ & $7.27 \pm 0.35$ & $8.32 \pm 0.88$ & 0.505 \\
\hline Cyclothymic & $6.62 \pm 0.32$ & $7.37 \pm 0.42$ & $5.06 \pm 1.31$ & 0.146 & $8.17 \pm 0.39$ & $7.61 \pm 0.56$ & $8.80 \pm 1.38$ & 0.601 \\
\hline Hyperthymic & $11.64 \pm 0.34$ & $11.36 \pm 0.44$ & $11.56 \pm 1.38$ & 0.879 & $10.28 \pm 0.39$ & $11.84 \pm 0.55$ & $10.64 \pm 1.36$ & 0.070 \\
\hline Irritable & $3.38 \pm 0.23$ & $3.99 \pm 0.30$ & $3.33 \pm 0.95$ & 0.266 & $3.57 \pm 0.29$ & $3.82 \pm 0.42$ & $5.27 \pm 1.04$ & 0.287 \\
\hline Anxious & $5.63 \pm 0.37$ & $5.67 \pm 0.48$ & $3.39 \pm 1.50$ & 0.339 & $6.92 \pm 0.46$ & $7.61 \pm 0.66$ & $9.84 \pm 1.63$ & 0.192 \\
\hline
\end{tabular}

SEM: standard error of mean, MANCOVA: multiple analyses of covariance 


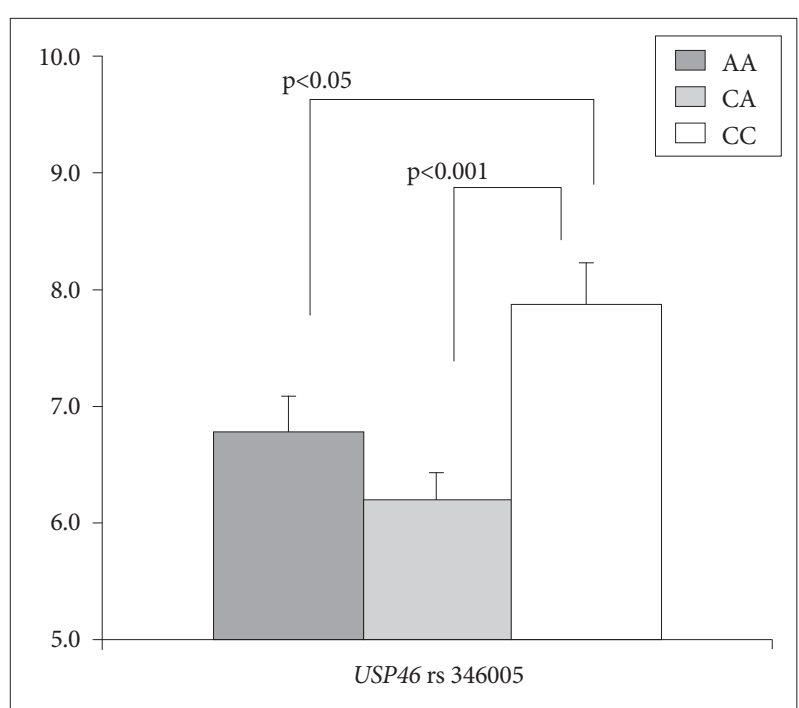

Figure 1. Post-hoc analysis comparing the TEMPS-A depressive subscale score for USP46 single-nucleotide polymorphism rs346005. Error bars indicate the standard error of mean. TEMPSA: Temperament Evaluation of the Memphis, Pisa, Paris, and San Diego Autoquestionnaire.

jects $\left(\mathrm{F}=7.79, \mathrm{p}<0.001, \eta \mathrm{p}^{2}=0.046\right)$. The association was not observed in female subjects $\left(\mathrm{F}=0.75, \mathrm{p}=0.472, \eta \mathrm{p}^{2}=0.007\right)$ (Table 2). In contrast, for rs2244291, no associations were found between the genotypes and the five affective temperaments, in males or females (Table 3).

Post-hoc comparisons showed that subjects who had the CC genotype (mean=7.89, $\mathrm{SD}=0.45$ ) at $\mathrm{rs} 346005$ showed a higher depressive temperament score than that of subjects with the AA genotype (mean $=6.80, \mathrm{SD}=0.30, \mathrm{p}<0.05$ ) and the CA genotype (mean $=6.18, \mathrm{SD}=0.21, \mathrm{p}<0.0001)$ in male subjects. There is no significant difference in the depressive temperament score between subjects with the AA genotype and subjects with the CA genotype $(\mathrm{p}=0.103)$ (Figure 1$)$.

\section{DISCUSSION}

We investigated the associations of the USP46 polymorphisms with the affective temperamental traits in healthy college students. The major finding was that the CC genotype of USP46 rs346005 was related to a higher depressive temperament score in male subjects. These findings suggest that affective temperamental traits are influenced by USP46.

Genetic associations between the ubiquitin proteasome system and affective temperament have not yet been reported. Although there is little direct evidence, our finding of the involvement of USP46 in depressive temperament is supported by indirect evidence. Theoretically, deubiquitinating enzymes, such as USP46, could play a role in regulating the stability of cellular protein and synaptic trafficking. ${ }^{25}$ Animal studies have shown that USP46-mutated mice exhibit a significantly shorter immobility time than wild type mice in a tail suspension test (TST), which indicates a change in depressive state. ${ }^{23}$ Furthermore, a genetic association study of major depression showed significant involvement of a USP46 haplotype in a Japanese population with major depressive disorder. ${ }^{21}$ In the study, rs346005 was the only SNP that showed signification association with major depressive disorder. Considering that a depressive temperament is a subclinical form of major depressive disorder, it is possible that USP46 may be involved in the pathophysiology of depression.

The biological mechanism underlying the association between USP46 and depressive temperament remains unclear. However, some possible explanations of the association can be proposed. First, USP46 may act as a mood regulator by modulating neurotransmitter systems, such as serotonin and GABA. The antidepressant imipramine, a tricyclic antidepressant that inhibits reuptake of monoamines, such as serotonin and norepinephrine, reduced immobility in the TST of USP46 mutated mice, ${ }^{23}$ which supports the monoamine hypothesis. ${ }^{26}$ In contrast, another study using USP46-knock-out mice reported that depressive behaviors in these mice were changed by administration of nitrazepam, GABAA receptor agonist, ${ }^{22}$ supporting the GABA hypothesis of major depressive disorder. ${ }^{27}$ Second, the ubiquitin system is known to regulate the circadian system by controlling circadian clock protein. ${ }^{28}$ Associations between the circadian system and affective temperament have previously been reported..$^{29,30}$ In a previous study, a depressive temperamental trait appeared to be associated with evening-type circadian preferences, along with cyclothymic, irritable, and anxious temperaments. ${ }^{30}$ Therefore, it can be assumed that USP46 influences affective temperamental traits by modulating the circadian system. Third, USP46 may affect the affective temperament by modulating synaptic plasticity. USP46 has been reported to play a role in regulating alpha-amino-3-hydroxy-5-methyl-4-isoxazole-propinic acid receptor (AMPAR), one of the key receptors involved in longterm potentiation and depression. ${ }^{25}$ USP46, which is known to be enriched at the neural synapse, may modulate brain function by deubiquitinating AMPAR. Because evidence supporting these speculations is lacking, further studies elucidating the pathways that may mediate the association between UPS and depressive temperament should be performed.

Unlike in male subjects, no main effects of USP46 polymorphisms were observed in female subjects. This finding could be explained in several ways. In general, gene expression and splicing is known to differ between males and females. ${ }^{31}$ From other perspectives, this gender difference might be related to an effect of sex hormones. Several studies have reported that the sex hormone estrogen modulates the ubiquitin systems, ${ }^{32,33}$ and plays an important role in synaptic plasticity. ${ }^{34}$ 
In this study, there was no difference in genotype distribution of rs346005 between males and females. This suggested that USP46 rs346005 is not the major cause of difference in depressive temperament between males and females. In addition, considering the fact that complex traits, such as affective temperaments, are medicated by many genes (not one gene) and their interactions, the higher score of depressive temperament in females seems to be affected by other conditions, such as sexual hormone and/or X-chromosome. We could not find other studies showing the sexual dimorphisms of USP46 rs346005, since there is only a few existing studies on USP46. However, we found several other evidence, although not USP46, suggesting genetic sexual dimorphisms on temperamental traits. Stankova et $a .^{35}$ reported that the sexual dimorphic effects of oxytocin receptor gene (OXTR) showed gender difference on harm avoidance; one of the aspects of temperamental traits. In our previous study of dopamine receptor D4 (DRD4) gene on affective temperament, the association between $\mathrm{DRD} 4$ polymorphism and affective temperament (cyclothymic and irritable) was found only in male subjects. ${ }^{13}$ In addition, since this study involved a small number of female subjects ( $n=217)$, the small sample size may not have been sufficient to obtain significant results.

There are several limitations in this study. First, most of our data were collected from college students, and it may not be generalizable. Although our sample can be considered a homogeneous group, with similar age, educational levels, and ethnicity, the results of this study should be considered cautiously. The results need to be replicated in other populations of different ages and ethnicities. Second, the present study was performed in a relatively small number of subjects. A further study in a larger sample size is needed to confirm the results. Third, we assessed only two SNPs. There are many more SNPs in USP46, and thus a more precise result could be obtained if additional SNPs were to be assessed. Fourth, affective temperamental scores could have been biased by social desirability, since TEMPS-A is a self-reporting scale. Fifth, we did not analyze gene-gene interaction and gene-environment interaction. Despite these limitations, this is the first study that reveals a possible genetic association between the UPS and affective temperaments.

In summary, the present study showed an association between a SNP in USP46 and depressive temperament. Our findings suggest that that some affective temperaments may be genetically predisposed to developing into affective disorders via vulnerable genes related to the UPS. In particular, USP46 may play a role in the pathophysiology of major depressive disorders by modulating a depressive temperament through dynamic proteolysis processes involving the UPS. Further research is required to elucidate influence of USP46 polymorphisms of affective temperaments in patients with mood disorders.

\section{Acknowledgments}

This study was supported by a grant from the Disease Oriented Translational Research through the Korea Health Industry Development Institute (KHIDI), South Korea (grant number: HI14C0202).

\section{REFERENCES}

1. Akiskal HS, Akiskal KK, Haykal RF, Manning JS, Connor PD. TEMPSA: progress towards validation of a self-rated clinical version of the Temperament Evaluation of the Memphis, Pisa, Paris, and San Diego Autoquestionnaire. J Affect Disord 2005;85:3-16.

2. Kawamura Y, Akiyama T, Shimada T, Minato T, Umekage T, Noda Y, et al. Six-year stability of affective temperaments as measured by TEMPSA. Psychopathology 2010;43:240-247.

3. Nigg JT. Temperament and developmental psychopathology. J Child Psychol Psychiatry 2006;47:395-422.

4. Kraepelin E. Manic-Depressive Insanity and Paranoia. Edinburgh: E. \& S. Livingstone; 1921.

5. Toda H, Inoue T, Tsunoda T, Nakai Y, Tanichi M, Tanaka T, et al. Affective temperaments play an important role in the relationship between childhood abuse and depressive symptoms in major depressive disorder. Psychiatry Res 2016;236:142-147.

6. Karam EG, Itani L, Fayyad J, Hantouche E, Karam A, Mneimneh Z, et al. Temperament and suicide: a national study. J Affect Disord 2015;184: 123-128.

7. Pompili M, Innamorati M, Gonda X, Serafini G, Sarno S, Erbuto D, et al. Affective temperaments and hopelessness as predictors of health and social functioning in mood disorder patients: a prospective follow-up study. J Affect Disord 2013;150:216-222.

8. Takeshima M, Oka T. Comparative analysis of affective temperament in patients with difficult-to-treat and easy-to-treat major depression and bipolar disorder: possible application in clinical settings. Compr Psychiatry 2016;66:71-78.

9. Vazquez GH, Gonda X, Zaratiegui R, Lorenzo LS, Akiskal K, Akiskal HS. Hyperthymic temperament may protect against suicidal ideation. J Affect Disord 2010;127:38-42.

10. Greenwood TA, Badner JA, Byerley W, Keck PE, McElroy SL, Remick RA, et al. Heritability and genome-wide SNP linkage analysis of temperament in bipolar disorder. J Affect Disord 2013;150:1031-1040.

11. Borkowska A, Bielinski M, Szczesny W, Szwed K, Tomaszewska M, Kalwa A, et al. Effect of the 5-HTTLPR polymorphism on affective temperament, depression and body mass index in obesity. J Affect Disord 2015;184:193-197.

12. Gonda X, Rihmer Z, Zsombok T, Bagdy G, Akiskal KK, Akiskal HS. The 5HTTLPR polymorphism of the serotonin transporter gene is associated with affective temperaments as measured by TEMPS-A. J Affect Disord 2006;91:125-131.

13. Kang JI, Namkoong K, Kim SJ. The association of 5-HTTLPR and DRD4 VNTR polymorphisms with affective temperamental traits in healthy volunteers. J Affect Disord 2008;109:157-163.

14. Landaas ET, Johansson S, Halmoy A, Oedegaard KJ, Fasmer OB, Haavik J. No association between the serotonin transporter gene polymorphism 5-HTTLPR and cyclothymic temperament as measured by TEMPS-A. J Affect Disord 2011;129:308-312.

15. Greenwood TA, Akiskal HS, Akiskal KK; Bipolar Genome S, Kelsoe JR. Genome-wide association study of temperament in bipolar disorder reveals significant associations with three novel Loci. Biol Psychiatry 2012;72:303-310.

16. Craney A, Rape M. Dynamic regulation of ubiquitin-dependent cell cycle control. Curr Opin Cell Biol 2013;25:704-710.

17. Hamilton AM, Zito K. Breaking it down: the ubiquitin proteasome sys- 
tem in neuronal morphogenesis. Neural Plast 2013;2013:196848.

18. Hegde AN, Haynes KA, Bach SV, Beckelman BC. Local ubiquitin-proteasome-mediated proteolysis and long-term synaptic plasticity. Front Mol Neurosci 2014;7:96.

19. Duman RS, Aghajanian GK, Sanacora G, Krystal JH. Synaptic plasticity and depression: new insights from stress and rapid-acting antidepressants. Nat Med 2016;22:238-249.

20. Wainwright SR, Galea LA. The neural plasticity theory of depression: assessing the roles of adult neurogenesis and PSA-NCAM within the hippocampus. Neural Plast 2013;2013:805497.

21. Fukuo Y, Kishi T, Kushima I, Yoshimura R, Okochi T, Kitajima T, et al. Possible association between ubiquitin-specific peptidase 46 gene and major depressive disorders in the Japanese population. J Affect Disord 2011;133:150-157.

22. Imai S, Mamiya T, Tsukada A, Sakai Y, Mouri A, Nabeshima T, et al. Ubiquitin-specific peptidase 46 (Usp46) regulates mouse immobile behavior in the tail suspension test through the GABAergic system. PLoS One 2012;7:e39084.

23. Tomida S, Mamiya T, Sakamaki H, Miura M, Aosaki T, Masuda M, et al. Usp46 is a quantitative trait gene regulating mouse immobile behavior in the tail suspension and forced swimming tests. Nat Genet 2009; 41:688-695

24. Vazquez GH, Tondo L, Mazzarini L, Gonda X. Affective temperaments in general population: a review and combined analysis from national studies. J Affect Disord 2012;139:18-22.

25. Huo Y, Khatri N, Hou Q, Gilbert J, Wang G, Man HY. The deubiquitinating enzyme USP46 regulates AMPA receptor ubiquitination and trafficking. J Neurochem 2015;134:1067-1080.

26. Hirschfeld RM. History and evolution of the monoamine hypothesis of depression. J Clin Psychiatry 2000;61(Suppl 6):4-6.
27. Luscher B, Shen Q, Sahir N. The GABAergic deficit hypothesis of major depressive disorder. Mol Psychiatry 2011;16:383-406.

28. Stojkovic K, Wing SS, Cermakian N. A central role for ubiquitination within a circadian clock protein modification code. Front Mol Neurosci 2014;7:69.

29. Chrobak AA, Tereszko A, Dembinska-Krajewska D, Arciszewska A, Siwek M, Dudek D, et al. Morningness-eveningness and affective temperaments assessed by the Temperament Evaluation of Memphis, Pisa and San Diego-Autoquestionnaire (TEMPS-A). Chronobiol Int 2017; 34:57-65.

30. Park CI, An SK, Kim HW, Koh MJ, Namkoong K, Kang JI, et al. Relationships between chronotypes and affective temperaments in healthy young adults. J Affect Disord 2015;175:256-259.

31. Trabzuni D, Ramasamy A, Imran S, Walker R, Smith C, Weale ME, et al. Widespread sex differences in gene expression and splicing in the adult human brain. Nat Commun 2013;4:2771.

32. Lai YJ, Liu L, Hu XT, He L, Chen GJ. Estrogen Modulates ubc9 Expression and Synaptic Redistribution in the Brain of APP/PS1 Mice and Cortical Neurons. J Mol Neurosci 2017;61:436-448.

33. Ogawa M, Yamaji R, Higashimura Y, Harada N, Ashida H, Nakano Y, et al. 17beta-estradiol represses myogenic differentiation by increasing ubiquitin-specific peptidase 19 through estrogen receptor alpha. J Biol Chem 2011;286:41455-41465.

34. Spencer-Segal JL, Tsuda MC, Mattei L, Waters EM, Romeo RD, Milner TA, et al. Estradiol acts via estrogen receptors alpha and beta on pathways important for synaptic plasticity in the mouse hippocampal formation. Neuroscience 2012;202:131-146.

35. Stankova T, Eichhammer P, Langguth B, Sand PG. Sexually dimorphic effects of oxytocin receptor gene (OXTR) variants on Harm Avoidance. Biol Sex Differ 2012;3:17. 